\title{
MULTIPLIER TRANSFORMATIONS ON COMPACT LIE GROUPS AND ALGEBRAS
}

\author{
BY
}

\author{
ROBERT S. STRICHARTZ (1)
}

ABSTRACT. Let $G$ be a semisimple compact Lie group and $T f=$ $\Sigma \phi(m) d_{m} \chi_{m} * f$ a bi-invariant operator on $L 2(G)$, where $\boldsymbol{X}_{m}$ and $d_{m}$ are the characters and dimensions of the irreducible representations of $G$, which are indexed by a lattice of points $m$ in the Lie algebra $\circlearrowleft$ in a natural way. If $\Phi$ is a bounded ad-invariant function on and

$$
\begin{aligned}
& \phi(m)=\Phi(m+\beta) \text { or } \\
& \phi(m)=\int_{G} \Phi(m+\beta-\operatorname{ad} g \beta) d g
\end{aligned}
$$

$\beta$ being half the sum of the positive roots, then various properties of $T$ are related to properties of the Fourier multiplier transformation on 4 with multiplier $\Phi$. These properties include boundedness on $L^{1}$, uniform boundedness on $L^{p}$ of a family of operators, and, in the special case $G=\mathrm{SO}(3)$, boundedness in $L^{p}$ for ad-invariant functions with $1 \leq p<3 / 2$. .

1. Introduction. Let $G$ be a compact group, and let $T$ be a bounded operator on $L^{2}(G)$ which commutes with left and right translation. Then there exists a bounded function $\phi(m)$ on $\hat{G}$, the space of equivalence classes of irreducible unitary representations of $G$, such that $T f=\Sigma \phi(m) d_{m} \chi_{m} * f$, where $\chi_{m}$ is the character and $d_{m}$ the dimension of the representation. A basic problem in harmonic analysis on $G$ is to relate properties of the function $\phi$ with properties of the operator $T$. We shall study this problem when $G$ is a semisimple compact Lie group.

Let $\&$ be the Lie algebra of $G$ and $B(x, y)$ the Killing form on $\&$. For any bounded measurable function $\Phi(x)$ on $\&$ we consider the corresponding Fourier multiplier operator

$$
f \rightarrow \int_{\Theta} \Phi(y) \hat{f}(y) e^{i B(x, y)} d y
$$

Received by the editors June 11, 1973.

AMS (MOS) subject classifications (1970). Primary 22E30, 43A75, 43A80, 42A18, 43A22; Secondary $43 A 35$.

Key words and phrases. Multiplier transformation, compact Lie group, bi-invariant operator, ad-invariant function on the Lie algebra.

(1) Research supported in part by NSF-GP-28251:

Copyright $\odot 1974$, American Mathematical Society 
where

$$
\hat{f}(y)=(2 \pi)^{-n} \int_{(y)} f(x) e^{-i B(x, y)} d y \text {. }
$$

If $\Phi$ is invariant under the adjoint representation of $G$ on $B$ then there is a close connection between this operator and the bi-invariant operators on $G$. To understand this connection we must describe how to pass from the function $\Phi$ to the function $\phi$. Now the irreducible unitary representations of $G$ are indexed by the dominant weights, which may be regarded as points in $\&$ via the Killing form. Thus the simplest relationship between $\Phi$ and $\phi$ would be to set

$$
\phi(m)=\Phi(m)
$$

For this to make sense we must have $\Phi$ continuous (or almost continuous), which we do not want to assume. A better relationship turns out to be

$$
\begin{aligned}
& \phi(m)=\Phi(m+\beta) \text { or even } \\
& \phi(m)=\int_{G} \Phi(m+\beta-\operatorname{ad} g \beta) d g
\end{aligned}
$$

where $\beta$ is half the sum of the positive roots.

Let op $(\phi)$ denote the bi-invariant operator on $G$ associated with $\phi$, and $\mathrm{Op}(\Phi)$ denote the Fourier multiplier operator on $\&$ associated with $\Phi$. We shall prove the following results.

(1) If $\mathrm{Op}_{\mathrm{p}}(\Phi)$ is bounded on $L^{1}(\&)$ then op $(\phi)$ is bounded on $L^{1}(G)$ under either $(*)$ or $(* *)$. In addition, under $(* *), \mathrm{op}(\phi)$ is positive definite if $\mathrm{OP}_{\mathrm{P}}(\Phi)$ is.

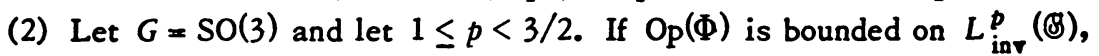
the ad-invariant $L^{p}$ functions on $\mathbb{Q}$, then under $(*)$ op $(\phi)$ is bounded on $L_{\text {inv }}^{p}(G)$, the central (conjugation invariant) functions in $L^{p}(G)$. The condition $1 \leq p<3 / 2$ implies that $\Phi$ is continuous herce $(*)$ is well defined. The group SO( 3$)$ is special because the exponential mapping is regular on an entire fundamental domain.

(3) Let $G$ and $p$ be arbitrary and denote $\Phi_{r}(x)=\Phi(x / r)$. The boundedness of $O_{\mathrm{p}}(\Phi)$ on $L^{p}(\mathbb{B})$ (or $L_{\mathrm{inv}}^{p}(\mathbb{B})$ ) is necessary for the uniform boundedness of op $\left(\phi_{r}\right)$ on $L^{p}(G)$ (or $L_{\text {inv }}^{p}(G)$ ) under $(*)$ or $(* *)$ as $r \rightarrow \infty$ (under $(*)$ we must assume $\Phi$ is continuous).

These results are stated for semisimple groups, but they may be extended with minor modifications to all compact Lie groups because they are trivial for the torus groups.

The proofs, which we present in the next two sections, rely heavily on two related formulas of Harish-Chandra [4]. The first (see (2.7) below) enables us to express the characters as ratios of Fourier transforms (see (2.8)). 
The idea behind Theorem 3 was used by Clerc ([1] and [2]) to study multipliers for spherical harmonic expansions. The problem of extending Theorem 1 to spherical expan sions on compact symmetric spaces would be interesting and difficult because no analogues of Harish-Chandra's formulas are known.

The ideal of relating multipliers on the Lie group and Lie algebra was inspired by the work of N. J. Weiss [6]. Weiss gives sufficient conditions for $L^{p}$ boundedness on $G, 1<p<\infty$, that are completely analogous to the MarcinkiewiczHörmander conditions for $\&$.

2. Sufficient conditions. Given a compact semisimple Lie group $G$ with Lie algebra $(8)$ we choose a maximal torus $T \subseteq G$ and a corresponding maximal abelian subalgebra $\mathcal{F}_{\mathcal{C}} \mathbb{B}$. We choose a set of positive roots $a_{1}, \cdots, a_{N}$ which we regard as elements of $\mathcal{F}_{2}$, and set $\beta=1 / 2\left(\alpha_{1}+\cdots+\alpha_{N}\right)$. We set

$$
\begin{aligned}
P(H) & =\prod_{j=1}^{N} B\left(H, a_{j}\right), \\
\Delta(H) & =\prod_{j=1}^{N} 2 i \sin 1 / 2 B\left(H, a_{j}\right), \text { for } H \in \mathcal{K}_{2}, \\
\Delta_{0}(H) & =\Delta(H) / P(H)
\end{aligned}
$$

where $B$ denotes the Killing form. We may extend $\Delta_{0}$ to \& by setting $\Delta_{0}(x)=$ $\Delta_{0}(H)$ for any $H \in \mathscr{F}_{2}$ in the same orbit as $x$ under the adjoint action of $G$ on $\mathbb{B}$. This definition is unambiguous because $\Delta_{0}(H)$ is invariant under the Weyl group.

Now let $\mu$ be any finite, ad-invariant measure on $\mathbb{B}$. We define two measures $\mu_{1}$ and $\mu_{2}$ on $G$ by

$$
\int_{G} f(g) d \mu_{1}(g)=\int_{M} f(\exp x) \overline{\Delta_{0}(x)} d \mu(x)
$$

and

$$
\int_{G} f(g) d \mu_{2}(g)=\int_{M} f(\exp x)\left|\Delta_{0}(x)\right|^{2} d \mu(x) .
$$

Note that these are finite, central measures on $G$, and that $\mu_{2}$ is positive if $\mu$ is positive. We shall show that, on the Fourier transform side, the mappings $\mu \rightarrow \mu_{1}$ and $\mu \rightarrow \mu_{2}$ correspond to $(*)$ and $(* *)$.

Lemma 1. Let $\Phi=\hat{\mu}=\int_{\Theta} e^{-i B(x, y)} d \mu(y)$. Then

$$
\int \overline{\chi_{m}(g)} d \mu_{1}(g)=\gamma^{\prime} d_{m} \Phi(m+\beta)
$$


and

$$
\int \overline{\chi_{m}(g)} d \mu_{2}(g)=\gamma^{\prime \prime} d_{m} \int_{G} \Phi(m+\beta-\operatorname{ad} g \beta) d g
$$

where $\gamma^{\prime}$ and $\gamma^{\prime \prime}$ are constants depending only on $G$.

Proof. The Weyl character and dimension formulas give

$$
\begin{aligned}
\chi_{m}(\exp H) & =\frac{\Sigma e^{i B(m+\beta, \sigma H)} \operatorname{det} \sigma}{\sum e^{i B(\beta, \sigma H)} \operatorname{det} \sigma}, \\
d_{m} & =P(m+\beta) / P(\beta)
\end{aligned}
$$

The sum is taken over the Weyl group. We use these in conjuction with a formula of Harish-Chandra [4, Theorem 2]

$$
P(H) P\left(H^{\prime}\right) \int_{G} e^{i B\left(\operatorname{ad} g H^{\prime}, H\right)} d g=\gamma \Sigma e^{i B\left(H^{\prime}, \sigma H\right)} \operatorname{det} \sigma
$$

(here $\gamma$ is a constant depending only on $G$ ) and the well-known $\Sigma e^{i B(\beta, \sigma H)} \operatorname{det} \sigma$ $=\Delta(H)$ to obtain

$$
\frac{\chi_{m}(\exp H)}{d_{m}}=\frac{P(\delta)}{\gamma} \frac{\int e^{i B(m+\beta, \text { ad } g H)} d g}{\Delta_{0}(H)} .
$$

But the same formula holds for any $x \in \mathbb{B}$ because both sides are ad-invariant. Thus from (2.1) we compute

$$
\begin{aligned}
\int_{G} \overline{\chi_{m}(g)} d \mu_{1}(g) & =\int_{\Theta} \overline{\chi_{m}(\exp x)} \overline{\Delta_{0}(x)} d \mu(x) \\
& =\gamma^{\prime} d_{m} \int_{G} \int_{\Theta} e^{-i B(\operatorname{ad} g(m+\beta), x)} d \mu(x) d g=\gamma^{\prime} d_{m} \hat{\mu}(m+\beta)
\end{aligned}
$$

because $\hat{\mu}$ is ad-invariant. From (2.2) we compute

$$
\begin{aligned}
\int_{G} \overline{\chi_{m}(g)} d \mu_{1}(g) & =\int_{M} \overline{\chi_{m}(\exp x)} \Delta_{0}(x) \overline{\Delta_{0}(x)} d \mu(x) \\
& =\gamma^{\prime \prime} d_{m} \int_{G} \int_{G} \int_{\Theta} e^{-i B\left(\operatorname{ad} g(m+\beta)_{0} x\right)} e^{i B\left(\operatorname{ad} g^{\prime} \beta x\right)} d \mu(x) d g d g^{\prime} \\
& =\gamma^{\prime \prime} d_{m} \int_{G} \int_{G} \hat{\mu}\left(\operatorname{ad} g(m+\beta)-\operatorname{ad}^{\prime} \beta\right) d g d g^{\prime} \\
& =\gamma^{\prime \prime} d_{m} \int_{G} \hat{\mu}(m+\beta-\operatorname{ad} g \beta) d g . \quad \text { Q.E.D. }
\end{aligned}
$$

Theorem 1. Let $\Phi$ be a bounded ad-invariant function on (8. If $\mathrm{Op}(\Phi)$ is bounded on $L^{1}(\Theta)$ then op $(\phi)$ is bounded on $L^{1}(G)$ where $\phi$ is given by $(*)$ or 
(**). If $\mathrm{Op}(\Phi)$ also preserves positivity (i.e., $\Phi$ is positive definite) then so does op $(\phi)$ provided.(**) is used.

Proof. It is well known that $O_{\mathrm{p}}(\Phi)$ is bounded on $L^{1}(\Phi)$ if and only if $\Phi=\hat{\mu}$ for a finite measure $\mu$, and $\mathrm{OP}_{\mathrm{p}}(\Phi)$ preserves positivity if and only if $\mu$ is a positive measure. If follows from the lemma that $\mathrm{op}(\phi)$ is convolution with $\mu_{1}$ or $\mu_{2}$ for $(*)$ or $(* *)$, and these operators obviously have the required properties. Q.E.D.

Remark. The positive definite multiplier transformations on any compact group may be characterized by the condition

$$
\sum \sum W\left(m, m^{\prime}, m^{\prime \prime}\right) d_{m} \phi(m) a\left(m^{\prime}\right) \overline{a\left(m^{\prime \prime}\right)} \geq 0
$$

for all finitely supported complex functions $a(m)$. Here $W$ is the generalized Clebsch-Gordon coefficient,

$$
W\left(m, m^{\prime}, m^{\prime \prime}\right)=\int_{G} \chi_{m}(g) \chi_{m^{\prime}}(g) \overline{\chi_{m^{\prime \prime}}(g)} d g,
$$

the number of times the representation $R_{m}$ occurs in $R_{m}^{*}, \otimes R_{m^{m}}$ We omit the proof of this assertion since it is a straightforward generalization of the proof of Bochner's theorem for compact abelian groups. It is also possible to prove part of Theorem 1 from this characterization and (2.8).

We next give an alternate description of the relation $(* *)$.

Lemma 2. There exists a bounded function $E(H)$ on 5 with support in the convex bull of the images of $\beta$ under the Weyl group, such that for any bounded ad-invariant function $\Phi(x)$ on 8 we bave

$$
\int_{G} \Phi(m+\beta-\operatorname{ad} g \beta) d g=d_{m}^{-1} \int_{S} \Phi(H) P(H) E(m+\beta-H) d H .
$$

If $Q$ denotes the square in $\mathbf{R}^{N}$ given by $Q=\left\{t:\left|t_{j}\right| \leq 1 / 2\right\}$ then

$$
\int_{G} \Phi(m+\beta-\operatorname{ad} g \beta) d g=\frac{c}{d_{m}} \int_{Q} \Phi(m+\beta-\alpha \cdot t) P(m+\beta-\alpha \cdot t) d t
$$

where $c$ is a constant depending only on $G$, and $a \cdot t=\Sigma t_{j} a_{j}$.

Proof. It suffices to prove (2.10) for

$$
\Phi(x)=\int_{G} e^{i B\left(\operatorname{ad} g^{\prime} x, y\right)} d g^{\prime}
$$

with $y \in \&$ arbitrary. For we may then obtain (2.10) in general by Fourier synthesis, and (2.9) then follows from the fact that the positive roots span $\mathfrak{F}_{\text {. }}$

Now using (2.7) we compute

$$
\begin{gathered}
\int_{G} \Phi(m+\beta-\operatorname{ad} g \beta) d g=\int_{G} e^{i B\left(\operatorname{ad} g^{\prime}(m+\beta), y\right)} d g^{\prime} \int_{G} e^{-i B(\operatorname{adg} \beta, y)} d g \\
=\frac{\gamma^{2}}{d_{m} P(\beta)^{2} P(y)^{2}} \sum \sum e^{i B\left(\sigma^{\prime}(m+\beta), y\right)} e^{-i B(\sigma \beta y)} \operatorname{det} \sigma \operatorname{det} \sigma^{\prime} .
\end{gathered}
$$


Also using (2.7) we compute

$$
\begin{aligned}
\int_{Q} \Phi(m & +\beta-\alpha \cdot t) P(m+\beta-\alpha \cdot t) d t=\gamma \int_{Q} \frac{\Sigma e^{i B(m+\beta-\alpha \cdot t, \sigma y)} \operatorname{det} \sigma}{P(y)} \mathrm{dt} \\
& =\frac{\gamma}{P(y)} \Sigma e^{i B(\sigma(m+\beta), y)} \operatorname{det} \sigma \int_{Q} e^{-i B(a \cdot t, \sigma y)} d t .
\end{aligned}
$$

Thus to establish (2.10) we need to show

$$
\int_{Q} e^{-i B(a \cdot t, \sigma y)} d t=\frac{c}{P(y)} \sum e^{-i B\left(\sigma^{\prime} \beta, y\right)} \operatorname{det} \sigma^{\prime}
$$

But $e^{-i B\left(a_{v}, \sigma_{y}\right)}=\Pi e^{-i t_{j} B\left(a_{j}, \sigma_{y}\right)}$ so the integral over $Q$ breaks up into the product on $N$ one-dimensional integrals which are easily computed:

$$
\int e^{-i B(a \cdot t, \sigma y)} d t=\prod \frac{e^{-i B\left(1 / 2 a_{j}, \sigma y\right)}-e^{+i B\left(1 / 2 a_{j}, \sigma y\right)}}{-i B\left(a_{j}, \sigma y\right)} .
$$

The numerator is well known to be $\Sigma_{\sigma^{\prime}} e^{-i B\left(\sigma^{\prime} \beta_{y} \sigma y\right)}$ det $\sigma^{\prime}$, while the denominator is clearly $(-i)^{N} P\left(\sigma_{y}\right)$. Since both are alternating under $\sigma$ we may drop it to obtain (2.11). Q.E.D.

We return now to (2.1) and (2.2). If $\mu$ is absolutely continuous with respect to Lebesgue measure, $d \mu=F(x) d x$, then $\mu_{j}$ is absolutely continous with respect to Haar measure, $d \mu_{j}=F_{j}(g) d g$. The relationships may be written

$$
\int_{G} f(g) F_{1}(g) d g=\int_{\Theta} f(\exp x) \overline{\Delta_{0}(x)} F(x) d x
$$

and

$$
\int_{G} f(g) F_{2}(g) d g=\int_{\Theta} f(\exp x)\left|\Delta_{0}(x)\right|^{2} F(x) d x .
$$

While $\left\|F_{j}\right\|_{1} \leq\|F\|_{1}$, the situation for $L^{p}$ norms requires clarification:

Lemma 3. Let $\&$ denote the integer lattice in 5 . Then

$$
F_{1}(\exp H)=c \sum_{8} F(H+2 \pi k) \Delta_{0}(H+2 \pi k)^{-1}
$$

and

$$
F_{2}(\exp H)=c \sum_{8} F(H+2 \pi k) .
$$

Furthermore $\left\|F_{j}\right\|_{p} \leq c\|F\|_{p}$ provided

$$
\sum_{q}\left|\Delta_{0}(H+2 \pi k)\right|^{j p^{\prime}-2} \in L^{\infty}, \text { wbere } 1 / p+1 / p^{\prime}=1 .
$$


Proof. From the familiar Weyl integration formula we have

$$
\int_{G} f(g) F_{1}(g) d g=c \int_{\mathrm{r}} f(\exp H) F_{1}(\exp H)|\Delta(H)|^{2} d H
$$

for any central function $f$, where $\Gamma=\left\{H \in \mathscr{F}_{2}:\left|H_{i}\right| \leq \pi\right\}$. A less familiar integration formula (see $[5$, p. 382]) states that

$$
\int_{\circlearrowleft} f(x) d x=\int_{\Theta} f(H)|P(H)|^{2} d H
$$

for any ad-invariant function $f$. Thus

$$
\begin{aligned}
\int_{\circlearrowleft} f(\exp x) \overline{\Delta_{0}(x)} F(x) d x \\
\quad=\int_{\circlearrowleft} f(\exp H) \overline{\Delta_{0}(H)} F(H)|P(H)|^{2} d H \\
\quad=\int_{\Gamma} \sum_{\nabla} f(\exp H) \overline{\Delta_{0}(H+2 \pi k)} F(H+2 \pi k)|P(H+2 \pi k)|^{2} d H
\end{aligned}
$$

and we read off (2.14) immediately. We establish (2.15) similarly.

Next assume (2.16) holds. Then

$$
\begin{aligned}
\left\|F_{j}\right\|_{p}^{p} & =c \int_{\mathbf{r}}\left|F_{j}(\exp H)\right|^{p}|\Delta(H)|^{2} d H \\
& \leq c \int_{\mathbf{\Gamma}}\left(\sum|F(H+2 \pi k)|\left|\Delta_{0}(H+2 \pi k)\right|^{j-2}\right)^{p}|\Delta(H)|^{2} d H .
\end{aligned}
$$

Now from Hölder's inequality we have

$$
\begin{aligned}
\sum_{Y} \mid & \left.F(H+2 \pi k)|| \Delta_{0}(H+2 \pi k)\right|^{j-2} \\
\leq & \left(\sum_{Y}|F(H+2 \pi k)|^{p}|P(H+2 \pi k)|^{2}\right)^{1 / p} \\
& \left.\cdot\left(\sum_{Y}\left|\Delta_{0}(H+2 \pi k)\right|^{(j-2) p^{\prime}}|P(H+2 \pi k)|^{-2 p^{\prime} / p}\right)\right)^{1 / p^{\prime}}
\end{aligned}
$$

Since $\Delta$ is periodic and $\Delta_{0}=\Delta / P$ we have

$$
\begin{gathered}
\left(\sum_{q}\left|\Delta_{0}(H+2 \pi k)\right|^{(j-2) p^{\prime}}|P(H+2 \pi k)|^{-2 p^{\prime} / p}\right)^{p / p^{\prime}}|\Delta(H)|^{2} \\
=\left(\sum_{q}\left|\Delta_{0}(H+2 \pi k)\right|^{j p^{\prime}-2}\right)^{p / p^{\prime}}
\end{gathered}
$$

which is bounded by (2.16). Thus 


$$
\begin{aligned}
\left\|F_{j}\right\|_{p}^{p} & \leq c^{\prime} \int_{\Gamma} \sum_{\boldsymbol{q}}|F(H+2 \pi k)|^{p}|P(H+2 \pi k)|^{2} d H \\
& \leq c^{\prime} \int_{\$}|F(H)|^{p}|P(H)|^{2} d H \leq c^{\prime}\|F\|_{p}^{p}
\end{aligned}
$$

by (2.17). Q.E.D.

Theorem 2. Let $G=S O(3)$, let $1 \leq p<3 / 2$ and let $\phi$ be given by (*). If $\mathrm{Op}(\Phi)$ is bounded on $L_{\mathrm{inv}}^{p}(\mathbb{(})$ then $\mathrm{op}(\phi)$ is bounded on $L_{\mathrm{inv}}^{p}(G)$.

Proof. In this case $\mathscr{F}_{2}$ is one dimensional, and $\Delta_{0}(H)=\sin 1 / 2 H / H$. Thus (2.16) holds whenever $j p^{\prime}-2>1$, which for $j=1$ means $1 \leq p<3 / 2$. Now let $f \in L_{\text {inv }}^{p}(G)$. We define $F \in L_{\text {inv }}^{p}(\&)$ by

$$
\begin{aligned}
F(H) & =f(\exp H) \Delta_{0}(H) \text { if }|H| \leq \pi, \\
& =0 \text { otherwise. }
\end{aligned}
$$

Note that

$$
\begin{aligned}
\|F\|_{p}^{p} & =\int_{-\pi}^{\pi}|F(H)|^{p}|P(H)|^{2} d H \\
& =\int_{-\pi}^{\pi}|f(\exp H)|^{p}\left|\Delta_{0}(H)\right|^{-2+p}|\Delta(H)|^{2} d H \leq c\|f\|_{p}^{p}
\end{aligned}
$$

because $\Delta_{0}$ is bounded away from zero on $[-\pi, \pi]$. Also

$$
\begin{aligned}
& \hat{F}(m+\beta)=\int_{\Theta} e^{-i B(m+\beta, x)} F(x) d x \\
& =\int_{\Theta} \int_{G} e^{-i B\left(m+\beta, \operatorname{adg} g^{\prime} x\right)} d g^{\prime} F(x) d x \\
& =\int_{\mathfrak{Q}} \int_{G} e^{-i B\left(m+\beta, \mathrm{ad}^{\prime} H\right)} d g^{\prime} F(H)|P(H)|^{2} d H \\
& =c \int_{-\pi}^{\pi} d_{m}^{-1} \overline{\chi_{m}(\exp H)} f(\exp H)|\Delta(H)|^{2} d H \\
& =c^{\prime} \int_{G} d_{m}^{-1} \overline{\chi_{m}(g)} f(g) d g
\end{aligned}
$$

by (2.17) and (2.8). Thus if $f=\Sigma_{a_{m}} \chi_{m}$ then $\hat{F}(m+\beta)=a_{m} / d_{m}$ and $\hat{F}(m+\beta) \Phi(m+\beta)=a_{m} \phi(m) / d_{m}$ by ${ }^{m}(*)$. But then Lemma 1 implies that $\left(\mathrm{OP}_{\mathrm{p}}(\Phi) F\right)_{1}$ $=c \Sigma \phi(m) a_{m} \chi_{m}=o p(\phi) f$. By Lemma $3\left\|\left(\mathrm{O}_{\mathrm{p}}(\Phi) F\right)_{1}\right\|_{p} \leq c\left\|\mathrm{OP}_{\mathrm{p}}(\Phi) F\right\|_{p}$, and by hypothesis $\left\|\mathrm{O}_{\mathrm{p}}(\Phi) F\right\|_{p} \leq c\|F\|_{p}$ so we have $\|\mathrm{op}(\phi) f\|_{p} \leq c\|f\|_{p^{*}}$ Q.E.D.

Remark. For the same reason $\mathrm{SO}(3)$ is special in another respect: All bounded operators on $L^{1}(G)$ are obtainable via Theorem 1 . 
3. Necessary conditions. Let $\Phi_{r}(x)=\Phi(x / r)$ and let $\phi_{r}$ be related to $\Phi_{r}$ by $(*)$ or $(* *)$. We assume that $\Phi$ is continuous when $(*)$ is used. We shall show how to obtain $\mathrm{Op}_{\mathrm{p}}(\Phi)$ by a limiting process from op $\left(\phi_{r}\right)$ as $r \rightarrow \infty$. If $F(x)$ has compact support on $\&$ we define $f_{r}(g)$ on $G$ for sufficiently large $r$ by setting

$$
f_{r}(\exp x / r)=F(x) \text { for } x \in \operatorname{support}(F)
$$

and letting $f_{r}$ vanish elsewhere. Let $n=\operatorname{dim} G$.

Lemma 4. There exists a constant $\gamma$ depending only on $G$, such that for all $F, F^{\prime}$ continuous with compact support on $\mathbb{B}$, we bave

$$
\lim _{r \rightarrow \infty} r^{n} \int_{G}\left[\mathrm{op}\left(\phi_{r}\right) f_{r}(g)\right] f_{r}^{\prime}(g) d g=\gamma \int_{\circlearrowleft}[\mathrm{Op}(\Phi) F(x)] F^{\prime}(x) d x
$$

Proof. Assume first that $\Phi$ decreases rapidly at infinity. Then we may interchange integrals to obtain

$$
\int_{\circlearrowleft}\left[\mathrm{OP}_{\mathrm{p}}(\Phi) F(x)\right] F^{\prime}(x) d x=\left(\frac{1}{2 \pi}\right)^{n} \int_{\circlearrowleft} \int_{\circlearrowleft}\left(\int_{\circlearrowleft} e^{i B(x-y, z)} \Phi(z) d z\right) F(y) F^{\prime}(x) d y d x
$$

On the other hand

$$
\begin{aligned}
r_{n} \int_{G} & \left.\operatorname{lop}\left(\phi_{r}\right) f_{r}(g)\right] f_{r}^{\prime}(g) d g \\
& =r^{n} \int_{G} \int_{G} \sum \phi_{r}(m) d_{m} \chi_{m}\left(g b^{-1}\right) f_{r}(b) f_{r}^{\prime}(g) d b d g \\
& =r^{n} \int_{M} \int_{M} \sum \phi_{r}(m) d_{m} \chi_{m}(\exp x \exp (-y)) f_{r}(\exp y) f_{r}^{\prime}(\exp x) \\
& \cdot\left|\Delta_{0}(x)\right|^{2}\left|\Delta_{0}(y)\right|^{2} d x d y \\
& =r^{-n} \int_{\circlearrowleft} \int_{M} \sum \phi_{r}(m) d_{m} \chi_{m}(\exp x / r \exp (-y / r)) \\
& \cdot F(y) F(x)\left|\Delta_{0}(x / r)\right|^{2}\left|\Delta_{0}(y / r)\right|^{2} d x d y .
\end{aligned}
$$

Thus to establish (3.1) it suffices to show

$$
\text { (3.2) } \lim _{r \rightarrow \infty} r^{-n} \sum \phi_{r}(m) d{ }_{m} \chi_{m}(\exp x / r \exp (-y / r))=\gamma \int_{\Theta} e^{i B(x-y, z)} \Phi(z) d z
$$

together with an estimate to justify the interchange of limit and integral.

We define $z_{r}$ by the equation $\exp x / r \exp (-y / r)=\exp z_{r} / r$, and note $\lim _{r \rightarrow \infty} z_{r}=x-y$. Next we make the change of variable $\lambda=m / r$, so that the 
left side of (3.2) becomes

$$
\lim _{r \rightarrow \infty} r^{-n} \sum \phi_{r}(r \lambda) \frac{P\left(\lambda_{r}+\beta\right)}{P(\beta)} \chi_{\lambda r}\left(\exp z_{r} / r\right) \text {. }
$$

Now $\lim _{r \rightarrow \infty} r^{-N} P(\lambda r+\beta)=P(\lambda)$ where $N=$ number of positive roots. Also

$$
\begin{aligned}
\lim _{r \rightarrow \infty} r^{-N} \chi_{\lambda r}\left(\exp z_{r} / r\right) \\
\quad=\lim _{r \rightarrow \infty} r^{-N} d_{\lambda r} \frac{\int e^{i B\left(\lambda r+\beta, \text { ad } g z_{r} / r\right)} d g}{\int e^{i B\left(\beta, \text { ad } g^{\prime} z_{r} / r\right)} d g^{\prime}} \\
\quad=P(\lambda) \int e^{i B(\lambda, \text { ad } g(x-y))} d g=\frac{c}{P(x-y)} \sum_{\sigma} e^{i B(\sigma \lambda, x-y)} \operatorname{det} \sigma .
\end{aligned}
$$

Since $P(\sigma \lambda)=P(\lambda)$ det $\sigma(3.3)$ becomes

$$
\lim _{r \rightarrow \infty} r^{2 N-n} c^{\prime} \sum \frac{P(\lambda)}{P(x-y)} e^{i B(\lambda, x-y)} \phi_{r}(r \lambda),
$$

where the summation extends over all $\lambda \in \mathscr{F}_{\mathcal{C}}$ such that $\lambda_{r}$ is a lattice point. Note that $n-2 N=\operatorname{dim} 5$.

Now if $\phi$ is given by $(*)$ then $\phi_{r}(r \lambda)=\Phi(\lambda+\beta / r)$ so that (3.4) is a Riemann approximation to the Riemann integral

$$
c^{\prime} \int_{\mathfrak{D}} \frac{P(H)}{P(x-y)} e^{i B(H, x-y)} \Phi(H) d H .
$$

But a formula of Harish-Chandra which is easily derivable from (2.7) (see [4, Theorem 3$]$ ) states that (3.5) is equal to

$$
c^{\prime \prime} \int_{\circlearrowleft} e^{i B(z, x-y)} \Phi(z) d z
$$

which establishes (3.2). To justify the interchange of integration and summation it suffices to show

$$
\sup _{r} \mid r^{-n} \sum \phi_{r}(r \lambda) d_{r \lambda} \chi_{r \lambda}(\exp x / r \exp (-y / r) \mid
$$

is bounded. But this follows from the fact that $\sup _{r} r^{-2 N} d_{r \lambda}\left\|\chi_{r \lambda}\right\|_{\infty}$ has polynomial growth in $\lambda$, which is an immediate consequence of the elementary estimate $\left\|\chi_{m}\right\|_{\infty} \leq d_{m}$.

If we use $(* *)$ we must modify the above reasoning slightly. Note that Lemma 2 assures us that $(* *)$ makes sense for bounde seasurable $\Phi$. By (2.9) we have

$$
\begin{aligned}
\phi_{r}(r \lambda) & =d_{r \lambda}^{-1} \int_{\$} \Phi_{r}(H) P(H) E(r \lambda+\beta-H) d H \\
& =P(\beta)_{r}^{n-2 N} \int_{\$} \Phi(H) \frac{P(r H)}{P(r \lambda+\beta)} E(r \lambda+\beta-r H) d H
\end{aligned}
$$


so that (3.4) becomes

$$
c^{\prime} \lim _{r \rightarrow \infty} \int \sum e^{i B(\lambda, x-y)} E(r \lambda+\beta-r H) \frac{P(\lambda) P(r H)}{P(x-y) P(r \lambda-\beta)} \Phi(H) d H .
$$

But the integrand converges to

$$
c e^{i B(H, x-y)} P(H) \Phi(H) / P(x-y),
$$

and the dominated convergence theorem applies to give (3.5). The remaining arguments for the $(*)$ case carry over.

Finally to remove the hypothesis that $\Phi$ decrease at infinity we multiply by $e^{-\epsilon B(x, x)}$ and let $\epsilon \rightarrow 0$. Q.E.D.

Theorem 3. A necessary condition for the boundedness of op $\left(\phi_{r}\right)$ on $L^{P}(G)$ (or $L \stackrel{\text { inv }}{p}(G)$ ) with norm independent of $r$ as $r \rightarrow \infty$ is that $\mathrm{Op}(\Phi)$ be bounded on

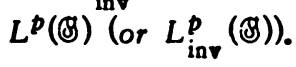

Proof. Note that $\lim _{r \rightarrow \infty} r^{n / p}\left\|f_{r}\right\|_{p}=c\|F\|_{p}$ because

$$
\begin{aligned}
r^{n}\left\|f_{r}\right\|_{p}^{p} & =r^{n} \int\left|f_{r}(\exp x)\right|^{p}\left|\Delta_{0}(x)\right|^{2} d x \\
& =\int|F(x)|^{p}\left|\Delta_{0}(x / r)\right|^{2} d x .
\end{aligned}
$$

Thus if $\|$ op $\left(\phi_{r}\right) f\left\|_{p} \leq c\right\| f \|_{p}$ for all $f \in L^{p}(G)$ (or $f \in L_{\text {inv }}^{p}(G)$ ) then from (3.1) we obtain

$$
\begin{aligned}
\gamma \int[\mathrm{Op}(\Phi) F(x)] F^{\prime}(x) d x & =\lim _{r \rightarrow \infty} r^{n} \int_{G}\left[\mathrm{op}\left(\phi_{r}\right) f_{r}(g)\right] f_{r}^{\prime}(g) d g \\
& \leq \lim _{r \rightarrow \infty} r^{n / p}\left\|_{\mathrm{op}}\left(\phi_{r}\right) f_{r}\right\|_{p^{r^{n}}}{ }^{n / p^{\prime}}\left\|f_{r}^{\prime}\right\|_{p^{\prime}} \\
& \leq c\|F\|_{p}\left\|F^{\prime}\right\|_{p^{\prime \prime}}
\end{aligned}
$$

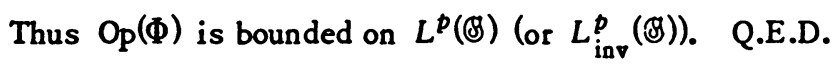

Note added in proof. R. Stanton has shown that Theorem 2 holds for $G=$ SU (2), and the analogue of Theorem 3 holds for all compact symmetric spaces.

\section{REFERENCES}

1. A. Bonami and J.-L. Clerc, Sommes de Cesàro et multiplicateurs des développements en harmoniques sphériques, Trans. Amer. Math. Soc., 183 (1973), 223-264.

2. J.-L. Clerc, Les sommes partielles de la décomposition en harmoniques sphériques ne convergent pas dans $L^{p}(p \neq 2)$, C. R. Acad. Sci. Paris Sér. A-B 274 (1972), A59-A61. MR $45 \# 2405$.

3. H. Freudenthal and H. DeVries, Linear Lie groups, Pure and Appl. Math., vol. 35, Academic Press, New York, 1969. MR 41 \#5546. 
4. Harish-Chandra, Differential operators on a semi-simple Lie algebra, Amer. J. Math. 79 (1957), 87-120. MR 18, 809.

5. S. Helgason, Differential geometry and symmetric spaces, Pure and Appl. Math., vol. 12, Academic Press, New York, 1962. MR 26 \#2986.

6. N. J. Weiss, $L^{p}$ estimates for bi-invariant operators on compact Lie groups, Amer. J. Math. 94 (1972), 103-118. MR 45 \#5278.

DEPARTMENT OF MATHEMATICS, CORNELL UNIVERSITY, ITHACA, NEV YORK 14850 